\title{
IMPLICACIONES DE LAS CONDICIONES DE LA GESTIÓN DEL CONOCIMIENTO Y EL APRENDIZAJE ORGANIZACIONAL SOBRE LOS RESULTADOS DE LA GESTIÓN. UN ESTUDIO COMPARATIVO
}

Implications of the Conditions of Knowledge Management and Organizational Learning on the Results of the Management. An Comparative Study

\section{RESUMEN}

Este estudio tiene como propósito identificar las condiciones de la gestión del conocimiento que favorecen el aprendizaje organizacional y que, a su vez, inciden en los resultados de la gestión. Además se propone un modelo general de análisis, contrastado a partir de un análisis comparativo de carácter exploratorio de una muestra de 1.251 empresas con más de 100 empleados de 33 países. Los resultados obtenidos muestran la comprobación de hipótesis derivadas de la revisión de la literatura y del modelo de relaciones propuesto.

Palabras clave: Gestión del conocimiento, aprendizaje organizacional, estudio comparativo.

\section{ABSTRACT}

This study aims to identify the enabling conditions of knowledge management that support organizational learning and, in turn, affect the results of management. It also suggests a general model of analysis, contrasted from an exploratory comparative analysis of a sample of 1.251 companies with over 100 employees in 33 countries. The results show the testing of hypotheses derived from literature review and model of proposed relationships.

Keywords: Knowledge management, organizational learning, comparative study. 
GESTIÓN DEL CONOCIMIENTO Y CAPACIDAD DE INNOVACIÓN

Modelos, Sistemas y Aplicaciones

\section{INTRODUCCIÓN}

En la sociedad del conocimiento, para que se mantengan competitivas, las organizaciones aprenden constantemente y dependen de conocimientos aplicados a los negocios. Como afirma Drucker (1999), no se puede negar que desde la mitad del siglo XX hubo una transición hacia este tipo de sociedad. La característica más importante de esta nueva organización social es que en ella el conocimiento pasa a ser el principal recurso para las personas y para la economía en general.

En este estudio se entiende la gestión de conocimiento como un proceso que amplía en ella misma el conocimiento creado por los empleados y los materializa en el nivel organizacional por medio del diálogo, comunicación, compartir experiencias y observaciones (Nonaka y Takeuchi, 1995). En otras palabras, se trata de la creación y desarrollo de condiciones que favorecen los stocks y flujos de aprendizaje, para obtener la eficiencia organizacional. Así pues, las organizaciones cuando dependen de conocimiento son llevadas a formar ambientes o condiciones, que presuponen procesos de aprendizaje y desarrollo.

A partir de esta reflexión, el estudio tiene como objetivo identificar las condiciones facilitadoras de la gestión del conocimiento que favorecen el aprendizaje organizacional y, que a su vez, inciden en los resultados de la gestión.

Dicho esto, la presencia y reconocimiento de stocks y flujos de conocimiento permiten generar condiciones que favorecen el aprendizaje organizacional. Este último es uno de los conceptos más amplios y de múltiples dimensiones dentro de la literatura de la organización de empresas. Significa esto que un elevado número de autores hacen referencia a él en sus trabajos e investigaciones, pues no existe una única definición ni de lo que es el aprendizaje ni de cómo se desarrolla dentro de la organización (Fiol y Lyles, 1985; Huber, 1991; Weick, 1991; DiBella y Nevis, 1998; Vera y Crossan, 2000). Estos estudios e investigaciones, mayormente, analizan el aprendizaje por sus efectos, reconociendo que es una condición para el cambio basado en el estado del conocimiento de una organización. Se tra- 
ta de una concepción que engloba los componentes cognitivos y de comportamiento vinculados al conocimiento (Argyris y Schön, 1978; Duncan y Weiss, 1979; Garvin, 1993; Senge, 1990; Walsh y Ungson, 1991; Crossan, Lane y White, 1999; Rant, 2001), según la cual el aprendizaje organizacional representa la transformación tanto del modo de pensar las cosas, como del modo de hacer las cosas. El resultado inmediato debe ser un conocimiento nuevo o mejorado, siendo el punto central del aprendizaje establecer una experimentación reactiva basada en la prueba y el error y, al mismo tiempo, hacer posible una resolución de problemas con carácter anticipativo.

De todo ello se deduce que todas las organizaciones deben comprometer una parte de sus recursos en el aprendizaje como parte de su desarroIlo, pues aquellas incapaces de hacer del aprendizaje una cualidad esencial, sencillamente, tienden a desaparecer (Nevis et al., 1995). Lo lógico es considerar que el aprendizaje constituye un comportamiento sistemático e inmanente a la misma (Duncan y Weiss, 1979). No es una elección, sino algo que forma parte de la definición de las organizaciones como sistemas complejos (Nicolini y Meznar, 1995; Alle, 1997). Así lo han reconocido numerosos autores (Levitt y March, 1988; Leonard Barton, 1993, 1995; DiBella, Nevis y Gould, 1996; Appelbaum y Reichart, 1997; Beveridge et al., 1997; DiBella y Nevis, 1998; entre otros), para quienes las organizaciones se comportan como sistemas de aprendizaje desarrollados espontáneamente en un determinado contexto. Por tanto, cuanto mayor sea la capacidad de aprendizaje del sistema, mayor será la probabilidad de alcanzar y mantener un alto resultado en la gestión en un entorno cambiante.

La metodología utilizada en este estudio es comparativa de carácter exploratorio, mediante una muestra de empresas de más de 100 empleados en 33 países, que permitirá contrastar el modelo general de relaciones propuesto, con el propósito de identificar las condiciones facilitadoras de la gestión del conocimiento percibidas por los encuestados que favorecen el aprendizaje organizacional y que, a su vez, inciden en los resultados de la gestión. 
GESTIÓN DEL CONOCIMIENTO Y CAPACIDAD DE INNOVACIÓN

Modelos, Sistemas y Aplicaciones

\section{FUNDAMENTACIÓN TEÓRICA}

En este estudio se considera la gestión del conocimiento como una herramienta fundamental que tiene por objeto garantizar la idoneidad del funcionamiento del conocimiento organizacional y que se materializa en unas condiciones facilitadoras, conformadas para amortiguar el alcance del dinamismo y las complejidades asociadas a mejorar la dotación de stocks y flujos de aprendizaje, y analizar su influencia en los resultados de la gestión.

A partir del análisis crítico de las principales aportaciones, planteamos nuestra propuesta de variables relativas a la gestión del conocimiento que influyen en el desarrollo del aprendizaje organizacional. En este sentido, la gestión del conocimiento es un concepto que presenta una dimensión que incluye condiciones de soporte y de los sistemas organizacionales básicos a partir de aspectos que favorecen los comportamientos que mejoran la consecución de los objetivos de la empresa.

Estas consideraciones nos conducen a analizar en qué medida es posible perfilar un modelo de gestión del conocimiento que, desde la teoría de recursos y capacidades (Barney, 1991; Peteraf, 1993; Grant, 1991, 1996; Amit y Schoemaker, 1993), concretamente, mediante el enfoque de capacidades dinámicas (Teece, et al., 1997; Eisenhardt y Martin, 2000), especifique cómo influyen una serie de condiciones facilitadoras sobre el aprendizaje organizacional. Con este propósito proponemos que la gestión del conocimiento habrá de materializarse en aquellas condiciones que permitan que las percepciones de los empleados den lugar a la aparición y el funcionamiento óptimo del conocimiento organizacional y que, por ende, intervienen positivamente en el aprendizaje organizacional.

El análisis presentado en este estudio toma como principal referencia las aportaciones que vinculan el estudio del aprendizaje a aspectos relacionados con la gestión y manejo del conocimiento en las organizaciones. Entre los autores que asocian estas variables, cabe distinguir varias corrientes: la centrada en la problemática de la creación de conocimiento en las organizaciones (Dodgson, 1993; Nonaka y Takeuchi, 1995; Sánchez y Heene, 1997; Nonaka e Ichijo, 1997; Nonaka, Reinmoeller y Senoo, 1998); la 
que se estudia la conversión del conocimiento individual en organizacional (Amponsem, 1991; Nonaka y Takeuchi, 1995); la focalizada en la adquisición de conocimiento por parte de la organización (Huber, 1991); la que vincula el aprendizaje organizacional a la creación de capacidades (Marengo, 1991; Andreu y Ciborra, 1994, 1995, 1996) y finalmente, la línea que relaciona el concepto de aprendizaje organizacional con el proceso de resolución de problemas (Andreu y Ciborra, 1994; Revilla, 1995; Andreu y Sieber, 1998).

Con base en las aportaciones mencionadas, este estudio se enmarca en la corriente que vincula el aprendizaje organizacional a la creación de capacidades (Marengo, 1991; Andreu y Ciborra, 1994, 1995, 1996). La creación de capacidades implica, asimismo, la creación de conocimiento. En concreto, se trata de competencias o capacidades nucleares que enlazan el aprendizaje organizacional con el proceso de resolución de problemas, como consecuencia de la ampliación de la base de conocimientos.

Como se ha señalado, en este estudio se analiza el sistema de relaciones propuesto entre gestión de conocimiento y aprendizaje organizacional desde el enfoque de capacidades dinámicas. Según Barney (2001), este enfoque es una versión evolucionista de la teoría de recursos y capacidades, cuyo principal interés consiste en estudiar cómo las capacidades de las empresas cambian en el tiempo, así como las implicaciones competitivas de estos cambios. El término capacidades dinámicas recoge básicamente la habilidad de las empresas para integrar, construir y reconfigurar las competencias internas y externas con el fin de responder rápidamente a los cambios del mercado (Teece, et al., 1997; Eisenhardt y Martin, 2000). Estos autores definen las capacidades dinámicas a partir de la concepción de los siguientes términos: por una parte, entienden que dinámicas se refiere a la capacidad para renovar competencias que logren la congruencia en un entorno de negocios cambiante y, capacidades recoge el papel clave de la dirección estratégica para adaptar, integrar y reconfigurar habilidades organizacionales internas y externas, recursos y competencias que permitan responder a las exigencias de los cambios del entorno. 
De acuerdo con Zollo y Winter (2002), una capacidad es un patrón aprendido y estable de la actividad colectiva mediante la cual la organización genera y modifica, sistemáticamente, sus rutinas operativas en la búsqueda de una eficacia mayor.

En este punto se intenta reorientar el concepto de aprendizaje para relacionar qué mecanismos están implicados en la creación y la evolución de las capacidades dinámicas, así como identificar qué rasgos distinguen una organización capaz de desarrollar sistemáticamente nuevas concepciones de las relaciones causales entre las acciones que esta toma y los resultados que obtiene en forma de rendimiento.

Por tanto, en este estudio se entiende el aprendizaje como una función dinámica de la organización resultado de la combinación de flujos y stocks de conocimiento, que favorecen la creación y desarrollo de nuevas capacidades organizacionales.

A los autores citados anteriormente, se suman Garvin (1993), Mayo y Lank (1994), Nevis, DiBella y Gould (1995), Probst y Büchel (1995) y Marquardt (1996), quienes realizan una reflexión sobre las condiciones organizacionales que favorecen el aprendizaje. Además de analizar cuál es el contexto estratégico que promueve el aprendizaje, también estudian cuál es el contexto organizacional que lo facilita. Estos autores destacan que los procesos de aprendizaje organizacional se ven favorecidos en el seno de marcos organizacionales caracterizados por la existencia de un equilibrio entre control y libertad, orden y desorden, estabilidad y cambio, así como entre centralización y descentralización. Estas condiciones son las que permiten, precisamente, crear espacios de innovación y creatividad dentro de la organización. Espacios donde se promueva la reflexión sobre las tareas, procesos de trabajo y modos de hacer, a fin de interrogarse sobre los mismos y, como resultado, aprender y mejorar.

En este sentido, la función dinámica del aprendizaje organizacional se ve materializada a partir de condiciones que favorecen su actuación y desarrollo. Dichas condiciones deberían estar presentes en el contexto orga- 
nizacional con el propósito de favorecer y mejorar los flujos de información y conocimiento entre los miembros de la empresa, que a su vez, permitiría ampliar la base de conocimiento, y además, altos niveles de aprendizaje.

Tomando como referencias el enfoque de capacidades dinámicas y la revisión de la literatura sobre la gestión del conocimiento y el aprendizaje organizacional, se desprenden las siguientes condiciones facilitadores: liderazgo (Garratt, 1987, 1990; Senge, 1990; McGill, Slocum y Lei, 1992; Mayo y Lank, 1994), comunicación (Nonaka y Takeuchi, 1995; Leonar-Barton, 1995; Nonaka, Toyama y Konno, 2001; Gold, Malhotra y Segars, 2001; Benavides y Quintana, 2003), colaboración (Daft y Weick, 1984; Fiol y Lyles, 1985; Nevis, et al., 1995; Nahapiet y Goshal, 1998; Scott, 2000), y compromiso (DiBella, et al., 1996, 1998; Handy, 1995; Kim y Mauborgne, 1997; Ulrich, 1998; Mowday, 1998; Cross y Baird, 2000; Bueno, 2001; Janz y Prasarnphanich, 2003).

Identificadas las condiciones de la gestión del conocimiento que favorecen el aprendizaje organizacional, se propone el modelo general de análisis.

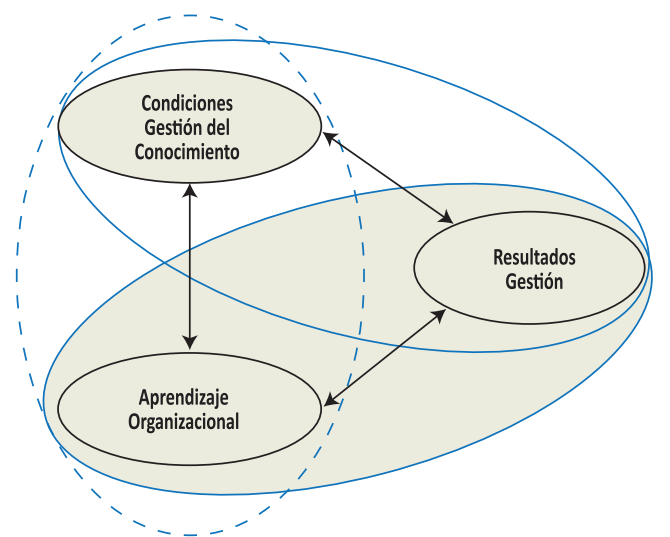

Figura 1. Modelo general de análisis

Fuente: Elaboración propia

El modelo tiene por objetivo comprobar las condiciones facilitadoras de la gestión del conocimiento identificadas en la literatura con las percep- 
ciones de los empleados encuestados. Una vez realizada la comprobación de las condiciones facilitadoras se debe determinar si estas favorecen el aprendizaje organizacional, y a su vez, los resultados de la gestión.

Si bien las referencias revisadas proponen hipótesis, modelos explicativos de las dinámicas organizativas e instrumentos empíricos de trabajo, dejan para futuras investigaciones la comprobación empírica de las hipótesis y de los modelos, con los instrumentos empíricos sugeridos (Fiol, 1991; Fiol y Huff, 1992; Reger, et al., 1994). Esta situación permitió definir las siguientes hipótesis:

H1: Las condiciones facilitadoras de la gestión del conocimiento -liderazgo, comunicación, colaboración y compromiso- influyen positivamente en el aprendizaje organizacional.

H2: El efecto positivo de las condiciones facilitadoras de la gestión del conocimiento sobre el aprendizaje organizacional está estrechamente relacionado con los resultados de la gestión, que se materializan en confianza y satisfacción.

\section{METODOLOGÍA}

\subsection{Características del estudio}

La metodología utilizada en este estudio es de carácter exploratorio, que de acuerdo con Selltiz, et al. (1987), propicia una mayor aproximación del investigador con el fenómeno del estudio, resultando, muchas veces, en una nueva comprensión de él. La propuesta es comprobar el modelo general de relaciones propuesto, a partir de la identificación de condiciones facilitadoras de la gestión del conocimiento que favorecen el aprendizaje organizacional, y que a su vez, inciden en los resultados de la gestión, validando también la aplicación del modelo general mediante un análisis comparativo por países.

Para ello, se realizó una investigación de levantamiento de datos, cuya estrategia es estudiar fenómenos que influyen en las interacciones entre 
personas y su contexto laboral (Selltiz, et al., 1987). Así, datos de toda o parte de una población deben ser recolectados con la finalidad de evaluar la incidencia relativa, distribución e interrelaciones de fenómenos (Kerlinger, 1964). Esta metodología fue adoptada para realizar el levantamiento sobre las condiciones facilitadoras de la gestión del conocimiento en las empresas involucradas.

\subsection{Objeto de estudio}

El estudio se realizó con base en la encuesta aplicada por GREAT PLACE TO WORK ${ }^{\circledR}$ de tipo survey, integrada por 58 ítems, para responder en una escala tipo Likert de cinco puntos. El propósito de esta encuesta fue analizar los fenómenos que influyen entre las interacciones, actitudes y percepciones de los empleados; se aplicó entre los meses de enero y diciembre de 2010 en 1.251 empresas, con más de 100 empleados, de 33 países. El muestreo se realizó por conveniencia, es decir, se eligieron las mejores empresas para trabajar en cada país. Las encuestas se realizaron a los empleados y fueron totalizadas en 480.291 respuestas válidas.

De los 58 ítems propuestos, 34 fueron considerados oportunos para efectos de este estudio, ya que influyen en las percepciones que tienen los empleados acerca de las condiciones facilitadoras del aprendizaje organizacional.

\subsection{Procedimientos metodológicos}

Para la comprobación de hipótesis, en primer lugar se realizó un análisis factorial con el software estadístico SPSS, cuyo propósito principal es definir la estructura subyacente en la matriz de datos y aborda el problema de cómo analizar la estructura de las interrelaciones entre un gran número de variables con la definición de una serie de dimensiones subyacentes comunes, conocidas como factores. Con el análisis factorial, se pueden identificar primero las dimensiones separadas de la estructura y determinar el grado en que se justifica cada variable por cada dimensión. Una vez que se determinan estas dimensiones y la explicación de cada variable, se 
pueden lograr los dos objetivos principales de este análisis, el resumen y la reducción de datos. Con el análisis factorial se obtienen dimensiones que, cuando son interpretadas y comprendidas, describen los datos con un número de conceptos mucho más reducido que las variables individuales originales, y entonces se puede sustituirlos por los ítems iniciales (Hair, et al., 2001; Luque, 2000).

Las técnicas analísticas de factores pueden lograr sus propósitos desde una perspectiva tanto exploratoria como confirmatoria: en este estudio, las dos fueron necesarias. A pesar de que muchos investigadores consideran el análisis factorial únicamente exploratorio, cabe destacar que las técnicas de análisis factorial extraen lo que proporcionan los datos y no tienen ninguna restricción a priori sobre la estimación de los componentes o el número de componentes a ser extraído. No obstante, en otras situaciones se tienen posicionamientos preconcebidos sobre la estructura real de los datos que se basan en un apoyo teórico o en un análisis factorial exploratorio realizado anteriormente. En estos casos, se requiere un análisis factorial que adopte un enfoque confirmatorio, es decir, valorar hasta qué punto los datos se ajustan a la estructura esperada (Hair, et al., 2001; Uriel y Aldás, 2005).

En segundo lugar, se realizó un análisis de conglomerados cuyo principal propósito es agrupar objetos basándose en las características que poseen, clasificando objetos de tal forma que cada uno sea muy parecido a los que hay en el conglomerado con respecto a algún criterio de selección predeterminado. Los conglomerados de objetos resultantes deberían mostrar un alto grado de homogeneidad interna y un alto grado de heterogeneidad externa (Hair, et al., 2001).

El valor teórico del análisis de conglomerados es el conjunto de variables que representan las características utilizadas para comparar objetos en ese análisis: dado que el valor teórico del análisis de conglomerados incluye solo las variables utilizadas para comparar objetos, determina su carácter. Esta es la única técnica multivariante que no estima el valor teórico empíricamente sino que utiliza el valor teórico especificado por el investigador. Este tipo de análisis es muy útil cuando se desean desarrollar 
las hipótesis concernientes a la naturaleza de los datos o para examinar las hipótesis previamente establecidas. Sin embargo, ese tipo de análisis puede caracterizarse como descriptivo y no inferencial, porque no tiene bases estadísticas sobre las cuales deducir inferencias estadísticas para una población a partir de una muestra, y se utiliza fundamentalmente como una técnica exploratoria. Finalmente, la solución de los conglomerados es totalmente dependiente de las variables utilizadas como base para medida de similitud (Johnson, 1998).

Seleccionadas las variables, se procedió a realizar el análisis de conglomerados siguiendo la aproximación de emplear una combinación de métodos jerárquicos. Se elegió el método de Ward con objeto de minimizar las diferencias internas de cada conglomerado y evitar los problemas de encadenamiento de las combinaciones iniciales inadecuadas, que tiene el método del vecino más cercano (Hair, et al., 2001).

\section{RESULTADOS}

Uno de los principales hallazgos fue la comprobación del modelo general de relaciones propuesto, ya que se puede afirmar la existencia de condiciones facilitadoras de la gestión del conocimiento -liderazgo, comunicación, colaboración y compromiso-, que tienen efecto positivo sobre el aprendizaje organizacional, y a su vez, en los resultados de gestión, y que son medidos por la confianza y la satisfacción en el trabajo. 
Tabla 1. Análisis factorial

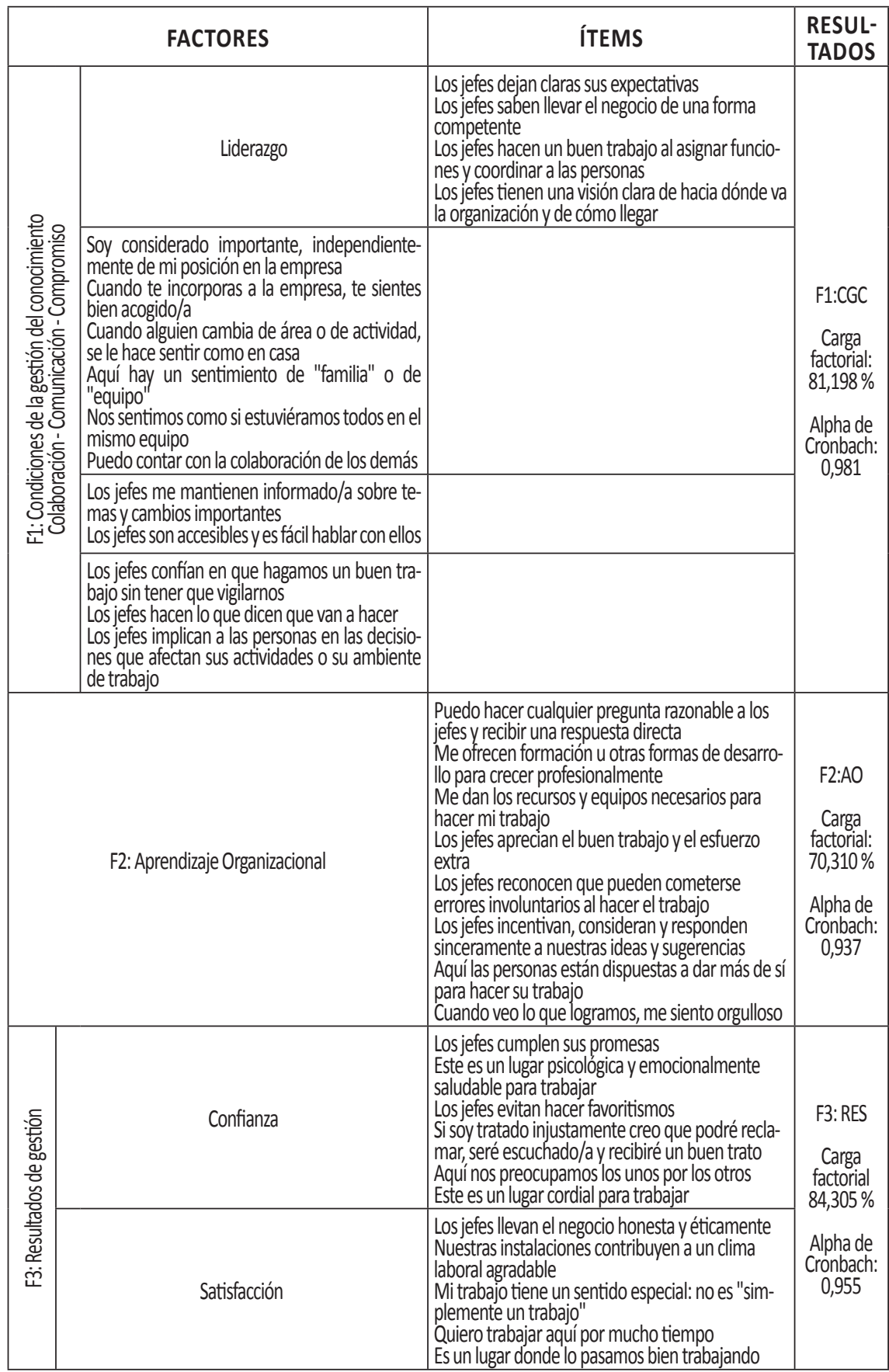


El número de ítems inicial utilizado en la encuesta fue de 55; para efecto de este estudio se realizó con 34 ítems, todos relacionados con el objeto de estudio como se describe en la Tabla 1 . Basados en el modelo de relaciones propuesto, el análisis factorial arrojó los siguientes resultados: Factor 1 (F1) Condiciones de la gestión del conocimiento (CGC), integrado por 15 ítems, confirma la presencia de las condiciones facilitadoras identificadas en la literatura -liderazgo, colaboración, comunicación y compromiso-, con una carga factorial de 81,198 \% y alpha de Cronbach de 0,981. Factor 2 (F2) Aprendizaje organizacional (AO), con 8 ítems, confirma la presencia de procesos de aprendizaje a nivel organizacional mediante el cual se integran conocimientos, habilidades y actitudes para conseguir cambios o mejoras para los empleados. Por lo tanto, el aprendizaje es una acción que toma el conocimiento como input y genera nuevo conocimiento. Este factor presenta una carga factorial de 70, 310 \% y alpha de Cronbach de 0,937.

El Factor 3 (F3) agrupa 11 ítems relativos a los resultados de la gestión. En la identificación de los resultados de la gestión se observó una desagregación en las percepciones de los empleados encuestados, ya que el Factor 3 identifica elementos de confianza y satisfacción en el trabajo, con una carga factorial de $84,337 \%$ y alpha de Cronbach de 0,955.

De los resultados obtenidos, se puede decir que en todos los casos, es decir, los cuatro factores resultantes, tanto las cargas factorial como alpha de Cronbach son significativos para el estudio.

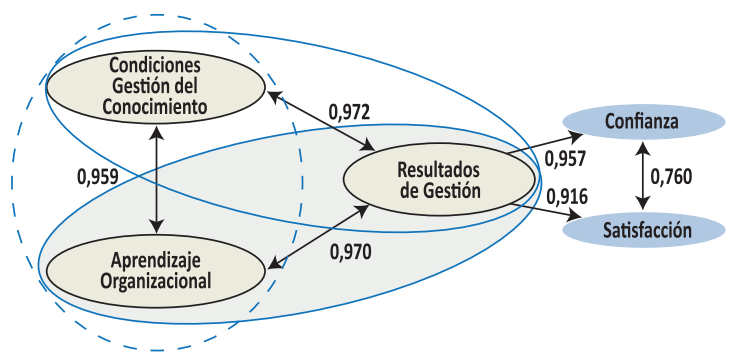

Figura 2. Modelo específico de análisis

Fuente: Elaboración propia 
GESTIÓN DEL CONOCIMIENTO Y CAPACIDAD DE INNOVACIÓN

Modelos, Sistemas y Aplicaciones

Además, la Tabla 2 que muestra la correlación de factores CGC, AO, RES presenta valores significativos y confirma el efecto positivo de la relación propuesta en el modelo de general de análisis.

Tabla 2. Correlación de factores

\begin{tabular}{|l|l|l|l|}
\hline \multicolumn{1}{|c|}{ FACTORES } & CGC-CONDICIONES & AO-APRENDIZAJE & RES-RESULTADOS \\
\hline CGC-Condiciones & & & \\
\hline AO-Aprendizaje & 0,959 & & \\
\hline RES-Resultados & 0,972 & 0,970 & \\
\hline
\end{tabular}

Por su parte, la aplicación del análisis de conglomerados permitió agrupar y comparar los 33 países en función de los factores previamente obtenidos. Como ya fue mencionado, el análisis se realizó con el método jerárquico de Ward (ver Tabla 3), teniendo en cuenta que el número de conglomerados ideal es 5. También, la Tabla 4 muestra los promedios de los grupos de conglomerados por factores, así como la desagregación de los resultados de gestión en los promedios obtenidos para los elementos confianza y satisfacción en el trabajo, considerados ambos oportunos para la comprensión de la correlación del modelo propuesto.

Tabla 3. Conglomerados obtenidos de países

\begin{tabular}{|c|l|}
\hline CONGLOMERADO & \multicolumn{1}{|c|}{ PAÍSES } \\
\hline Grupo 1 & Perú, Colombia, Dinamarca, Estados Unidos, Canadá, Australia \\
\hline Grupo 2 & Suecia, Reino Unido, Irlanda, Finlandia \\
\hline Grupo 3 & $\begin{array}{l}\text { Paraguay, México, Venezuela, Ecuador, Bolivia, Holanda, Portugal, India, } \\
\text { Brasil, Uruguay, Suiza, Noruega, Alemania, Austria }\end{array}$ \\
\hline Grupo 4 & Japón, Polonia, Francia, Bélgica \\
\hline Grupo 5 & España, Chile, Italia, Grecia, Argentina \\
\hline
\end{tabular}

Tabla 4. Promedios de los 5 grupos de conglomerados por factores

\begin{tabular}{|c|c|c|c|c|c|}
\hline PROMEDIOS & $\begin{array}{c}\text { CGC- } \\
\text { CONDICIONES }\end{array}$ & $\begin{array}{c}\text { AO- } \\
\text { APRENDIZAJE }\end{array}$ & RES-RESULTADOS & CF-CONFIANZA & $\begin{array}{c}\text { ST- } \\
\text { SATISFACCIÓN }\end{array}$ \\
\hline Grupo 1 & 86.2643 & 85.5841 & 87.3099 & 86.0741 & 88.7928 \\
\hline Grupo 2 & 79.4944 & 79.4538 & 80.8807 & 82.2577 & 79.2284 \\
\hline Grupo 3 & 81.1815 & 80.7665 & 83.1074 & 80.5092 & 86.2252 \\
\hline Grupo 4 & 66.5268 & 67.5934 & 68.3432 & 65.0677 & 72.2738 \\
\hline Grupo 5 & 73.3447 & 72.8243 & 74.5402 & 72.0680 & 77.5068 \\
\hline
\end{tabular}


- Grupo 1: Perú, Colombia, Dinamarca, Estados Unidos, Canadá, Australia

Este grupo presenta los índices más altos en todos los factores. Se observa que el índice de resultado de gestión -satisfacción en el trabajo- es el más alto de todos los valores, es decir, la percepción de la satisfacción es muy positiva. Sin embargo, es un grupo heterogéneo sin una característica común, lo que imposibilita encontrar algo que explicaría el efecto positivo. Obtener índices de percepción superiores a 85 en el promedio es un gran desafío, pues la muestra de empresas de los países agrupados deberían ser estudiadas con mayor atención para conocer si los resultados obtenidos obedecen a estrategias de empresas globales o condiciones propias de los países, que se derivan de políticas impulsoras que favorecen la competitividad de las empresas, mediante el desarrollo de procesos de aprendizaje y buenas prácticas, que también generan resultados relacionados a la confianza y la satisfacción en el trabajo.

- Grupo 2: Suecia, Reino Unido, Irlanda, Finlandia

Por contar con países bastante desarrollados, se supone que se encuentran empresas con altos niveles de compromiso hacia la generación de nuevo conocimiento y capacidad de aprendizaje. Es posible afirmar que las condiciones facilitadoras de la gestión del conocimiento que favorecen el aprendizaje, inciden en los resultados de gestión, y son bien percibidas a través de la satisfacción en el trabajo. Sin embargo, presenta especial atención el resultado de gestión basado en la confianza, ya que este es el factor con mayor índice. Además, es el único grupo en que la confianza representa la percepción más alta de los factores, lo que puede indicar modelos eficientes en la gestión en sus empresas.

- Grupo 3: Paraguay, México, Venezuela, Ecuador, Bolivia, Holanda, Portugal, India, Brasil, Uruguay, Suiza, Noruega, Alemania, Austria

A pesar de que el tercer grupo está integrado por países muy 
diversos -América Latina, Europa e India-, el punto en común son los altos índices de los factores, solo menores que los obtenidos en el primer grupo. Es posible decir que, relativamente, el factor satisfacción está bastante alto y sería la característica más fuerte de este conglomerado.

- Grupo 4: Japón, Polonia, Francia, Bélgica

Al igual que el grupo 2, el cuarto conglomerado está compuesto por países con alto nivel de desarrollo. Esto haría pensar que son países con buenas condiciones facilitadoras del aprendizaje. Sin embargo, en este grupo las valoraciones de los encuestados son más críticas al percibir y evaluar las condiciones facilitadoras que inciden sobre el aprendizaje. Es decir, que las condiciones facilitadoras son menos favorables sobre el aprendizaje. Todos los índices de los factores son relativamente bajos (en comparación con los otros grupos), y se observa que es el grupo que presenta la mayor diferencia entre los factores que identifican los resultados de la gestión: confianza y satisfacción. Así, la confianza es el factor peor percibido de todos los valores de la tabla comparativa.

- Grupo 5: España, Chile, Italia, Grecia, Argentina

Con una característica predominantemente latina, este grupo presenta índices medianos comparados con los otros grupos. Esto significa que en este contexto se perciben razonablemente bien las condiciones y el aprendizaje que conducen a los resultados de gestión, tanto confianza como satisfacción. Así como el grupo 4, posee un índice relativamente bajo para el factor confianza. No obstante, la valoración del factor satisfacción en el trabajo muestra el mayor índice en este grupo.

Finalmente, para validar el modelo se procedió a contrastar las hipótesis propuestas, a partir de la relación causal de variables agrupadas por bloques, como se describe en el siguiente epígrafe. 


\section{CONCLUSIONES Y DISCUSIÓN}

Una vez obtenido para cada variable latente un modelo de medida satisfactorio en las fases anteriores, procedemos al contraste de las hipótesis propuestas. Para lograr este fin, estudiamos los modelos de medida previamente estimados, resultados del proceso de depuración de las escalas. La estimación del modelo se ha realizado en tres bloques.

El primer bloque agrupa las condiciones de la gestión del conocimiento necesarias para que este concurra y evolucione dentro de la organización, a partir de los elementos -liderazgo, colaboración, comunicación y compromiso-, que inciden sobre el aprendizaje.

En el segundo bloque consideramos el aprendizaje organizacional como la principal variable dependiente del modelo. Este constituye uno de los elementos críticos para mantener a la organización en un estado de desarrollo y evolución permanentes, pues el aprendizaje es un comportamiento inherente a toda la organización, destinado a mejorar su capacidad de adaptación y anticipación a las exigencias del entorno. Como es mencionado en este estudio se entiende el aprendizaje organizacional como una capacidad dinámica en contacto permanente con el entorno que favorece la evolución continua que experimentan los conocimientos en la organización. Esto permite obtener una visión suficientemente completa del funcionamiento de la organización como sistema de aprendizaje, constituyendo una base que nos permite explicar las relaciones propuestas, mediante el modelo general de análisis.

El tercer bloque muestra, una vez determinado el efecto entre las condiciones de la gestión del conocimiento y el aprendizaje organizacional, la comprobación de cuáles son sus consecuencias efectivas sobre los resultados de la gestión.

Esta separación obedece, en el primer y segundo bloque, a nuestro deseo de contrastar la influencia específica de cada grupo de elementos, evitando cualquier interferencia entre los dos grupos de variables explicativas, cuya relación fue contrastada por separado, en atención a las exigencias 
metodológicas. Todo ello ha permitido comprobar la hipótesis uno (H1), al obtener coeficientes positivos y significativos de las variables correspondientes. Estos coeficientes confirman el efecto positivo de las condiciones facilitadoras de la gestión del conocimiento -liderazgo, colaboración, comunicación y compromiso- sobre el aprendizaje organizacional.

Una vez comprobada la relación propuesta en el primer y segundo bloque, el tercer bloque permitió conocer el carácter sistémico y dinámico del modelo de relaciones propuesto, pues establece la relación entre las condiciones de la gestión del conocimiento que favorecen el aprendizaje organizacional, y su incidencia sobre los resultados. Con base en este planteamiento y el análisis de los resultados obtenidos de los coeficientes positivos y significativos, a partir de los métodos estadísticos multivariantes utilizados, tanto análisis factorial como análisis de conglomerados, se comprueba la hipótesis dos ( $\mathrm{H} 2)$, que establece que el efecto positivo de las condiciones facilitadoras de la gestión del conocimiento sobre el aprendizaje organizacional está estrechamente relacionado con los resultados de la gestión, y estos se materializan en confianza y satisfacción en el trabajo.

Cabe mencionar que los resultados del análisis de conglomerados, muestran en los cinco grupos hallados, diferencias significativas en las percepciones y valoraciones de los empleados en cuanto a la confianza y satisfacción en el trabajo, como resultados de la gestión de las empresas.

En el ámbito académico, el estudio propone no solo la identificación de condiciones de la gestión del conocimiento que favorecen el aprendizaje organizacional, sino también el impacto que tienen estas en los resultados de la gestión de las empresas. Todo ello, a partir de la percepción y valoración de los empleados. Sin embargo, somos conscientes de las limitaciones del estudio al no ser generalizable en empresas de menor tamaño y la falta de sectorización, además de la ausencia de variables infraestructurales como el uso de tecnologías de información y comunicación, sistemas de vigilancia tecnológica, sistemas de decisión, estructura, etc., que podrían comprender más las valoraciones resultado de la encuesta. También, la au- 
sencia de datos financieros no permitió conocer cómo los resultados de la gestión, que se materializan en la confianza y la satisfacción en el trabajo inciden en los resultados económicos.

Sin duda, las limitaciones del estudio nos llevan a continuar avanzando en el análisis, tanto del comportamiento organizacional como de las formas de valoración de las empresas, pues la importancia de estas no está exclusivamente en el valor contable sino también en el valor de mercado y el conjunto de percepciones resultado de la relación con los clientes internos y externos.

\section{REFERENCIAS BIBLIOGRÁFICAS}

Alle, V. (1997). The Knowledge Evolution. Expanding Organizational Intelligence. Boston: Butterworth-Heinemann.

Amit, R. \& Schoemaker, P. (1993). Strategic Asset and Organizational Rent. Strategic Management Journal, 14, 33-46.

Amponsem, H. (1991). Organizational Learning through Internal Systems. Strategic Alliances and Networks. (Tesis doctoral). Queen's University at Kingston, Canadá.

Andreu, R. \& Ciborra, C. (1994). Information Systems for the Learning Organization. Speech delivered Technology and Organizational Change. Nijenrode University.

Andreu, R. \& Ciborra, C. (1995). The Role of IT in Creating an Effective Knowledge Base for the Learning Organization. EFMD Forum, 1.

Andreu, R. \& Ciborra, C. (1996). Core Capabilities and Information Technology: An Organisational Learning Approach. In: B. Moingeon, \& A. Edmondson. Organizational and Competitive Advantage. Londres: Sage.

Andreu, R. \& Sieber, S. (1998). Knowledge and Problem Solving: a Proposal for a Model of Individual and Collective Learning. Working Paper, 99/1. IESE.

Appelbaum, S. H. \& Reichart, W. (1997). How to Measure an Organization's 
Learning Ability: A Learning Orientation (part I). Journal of Workplace Learning, 7(9), 225-239.

Argyris, C. \& Schön, D. (1978). Organizational Learning: A Theory of Action Perspective. Reading, M.A.: Addison Wesley.

Barney, J.B. (2001). Resource-based theories of competitive advantage: a ten-year retrospective on the resource based view. Journal of Management, 27, 643-650.

Benavides, C. A. \& Quintana, C. (2003). Dirección del Conocimiento y Calidad Total. Madrid: Díaz de Santos.

Beveridge, M., Gear, A.E. \& Minkes, A. L. (1997). Organizational Learning and Strategic Decision Support. The Learning Organization, 5(4), 217-227.

Bueno, E. (2001). Creación, medición y gestión de intangibles: propuesta de modelo conceptual. En Formas y reformas de la nueva economía, Revista Madrid, monografía, 1, 43-48.

Cross, R. \& Baird, L. (2000). Technology Is Not Enough: Improving Performance by Building Organizational Memory. Sloan Management Review, Spring, 41, 69-78.

Crossan, M. M., Lane, H. W. \& White, R.E. (1999). An Organizational Learning Framework: from Intuition to Institution. Academy of Management Review, 3(24), 522- 537.

Daft, R. \& Weick, K. (1984). Toward a Model of Organization as Interpretation Systems. Academy of Management Review, (9), 284-295.

DiBella, A. J. \& Nevis, E. (1998). How Organizations Learn. San Francisco: Jossey Bass.

DiBella, A. J., Nevis, E. C. \& Gould, J. M. (1996). Understanding Organizational Learning Capability. Journal of Management Studies, 3(33), 361-379.

Dodgson, M. (1993). Organizational Learning: A Review of Some Literatures. Organizations Studies, (14), 375-394.

Drucker, P. F. (1999). Managing oneself. Harvard Business Review, 77(2), 64-74. 
Duncan, R. \& Weiss, A. (1979). Organizational Learning: Implications for Organizational Design. Research in Organizational Behaviors, (1), 75-123.

Eisenhardt, K. \& Martin, J. (2000). Dynamic Capabilities: The Evolution of Resources in Dynamic Markets. Strategic Management Journal, 21, 1105-1121.

Fiol, C. M. (1991). Managing culture as a competitive resource: An identity-based view of sustainable competitive advantage. Journal of Management, 17, 191-211.

Fiol, C. M. \& Huff, A. S. (1992). Map for Manager: Where are we? Where do we go from here? Journal of Management Studies, 29, 267-285.

Fiol, C. M. \& Lyles, M. A. (1985). Organizational Learning. Academy of Management Review, 4(10), 803-813.

Garratt, B. (1987). The Learning Organization: and the need for directors who think. Cambridge: Gower.

Garratt, B. (1990). Creating a Learning Organization: a guide to Leadershipnd, Learning and Development. Londres: Director Book.

Garvin, G. A. (1993). Building a Learning Organization. Harvard Business Review, July/August, 78-91.

Gold, A. H., Malhotra, A. \& Segars, A. H. (2001). Knowledge Management: an Organizational Capabilities Perspective. Journal of Management Information Systems, 1(18), 185-214.

Grant, R. M. (1991). The Resource-Based Theory of Competitive Advantages: Implications for Strategy Formulation. California Management Review, 114-135.

Grant, R. M. (1996). Prospering in Dynamically-Competitive Environments: Organizational Capability as Knowledge Integration. Organization Science, 7, 375-387.

Hair, J. F. Jr, Anderson, R. E., Tatham, R. L. \& Black, W. C. (2001). Análisis Multivariante. Madrid: Prentice Hall.

Handy, C. (1995). Trust and the Virtual Organization. Harvard Business Review, 3(73), 40-50. 
Huber, G. (1991). Organizational Learning: The Contributing Processes and the Literatures. Organization Science, (2), 88-115.

Janz, B. \& Prasarnphanich, P. (2003). Understanding the antecedents of effective knowledge management. The importance of a knowledge-centered culture. Decision sciences, 34(2), 351-385.

Johnson, D. E. (1998). Applied Multivariance Methods for Data Analysts. Nueva York: Brooks Cole Publishing Company.

Kerlinger, F. N. (1964). Foundations of Behavioral Research: Educational and Psychological Inquiry. New York: John Wiley.

Kim, W. C. \& Mauborne, R. (1997). Fair Processes: Managing in the Knowledge Economy. Harvard Business Review, July/August, 65-75.

Leonard Barton, D. (1993). La fábrica como laboratorio de aprendizaje. Harvard Deusto Business Review, 58, 46-61.

Leonard Barton, D. (1995). Wellsprings of Knowledege: Building and Sustaining the Sources of Innovation. Boston: Harvard Business School Press.

Levitt, B. y March, J. (1988). Organizational Learning. American Review of Sociology, (14), 24-39.

Luque, T. (2000). Técnicas de análisis de datos en investigación de mercados. Madrid: Pirámide.

Marengo, L. (1991). Knowledge, Coordination and Learning in an Adaptive Model of Firm. (Tesis doctoral). Sussex University, Sussex.

Marquardt, M. J. (1996). Building the Learning Organization. Londres: McGraw-Hill.

Mayo, A. \& Lank, E. (1994). The Power of Learning. Institute of Personnel and Development. Londres, London.

McGill, M. E., Slocum, J. W. \& Lei, D. Jr. (1992). Management Practices in Learning Organizations. Organization Dynamics, Summer, 5-17.

Mowday, R. (1998). Reflections on the study and relevance of organizational commitment. Human Resource Management Review, 8(4), 387-401.

Nahapiet, J. \& Goshal, S. (1998). Social Capital, Intellectual Capital and the 
Organizational Advantage. The Academy of Management Review, 2(23), 242-266.

Nevis, E., Dibella, A. \& Gould, J. (1995). Understanding organizations as learning systems. Sloan Management Review, Winter, 73-85.

Nicolini, D. \& Meznar, M. B. (1995). The Social Construction of Organizational Learning: Conceptual and Practical Issues in the Field. Human Relations, 7(48).

Nonaka, I. \& Ichijo, K. (1997). Creating Knowledge in the Process Organization. In J. P. Walsh \& J. S. Huff (eds). Advances in Strategic Management, (14), 45-52.

Nonaka, I., Toyama, R. \& Konno, N. (2001). SECl, Ba and Leadership: a unified model of dynamic knowledge creation. In, I. Nonaka \& D. Teece (eds.). Managing Industrial Knowledge: creation, transfer, and utilization (pp.13-43). Londres: Sage.

Nonaka, I. \& Takeuchi, H. (1995). The knowledge-creating company. Oxford: Ed, Oxford University Press.

Nonaka, I., Reinmoeller, P. \& Senoo, D. (1998). Management Focus; the "ART" of Knowledge: Systems to Capitalize on Market Knowledge. European Management Journal, 16(6), 673-684.

Peteraf, M. (1993). The Cornerstones of Competitive Advantage. A Resource-Based View. Strategic Management Journal, 14, 179-191.

Probst, G. \& Büchel, B. (1995). La Practique de l'Entreprise Apprenante. Les Éditions d'Organosation. París: Editions d'Organisation

Rant, M. (2001). The Role of Organization in Knowledge Management: The Empirical Study of the Organization as the Focal Knowledge Creation and Sharing Mechanism. Second European Conference on Knowledge Management, Bled, Slovenia.

Reger, R. K., Gustafson, L. T., Demarie, S. M., \& Mullane, J. V. (1994). Reframing the organization: Why implementing total quality is easier said than done. Academy of Management Review, 19, 565-584.

Revilla, E. (1995). Factores determinantes del aprendizaje organizativo: un 
modelo de desarrollo de productos (Tesis doctoral). España: Universidad de Valladolid.

Sánchez, R. \& Heene, A. (1997). Strategic Learning and Knowledge Management. Nueva York: John Wiley and Sons.

Scott, J. E. (2000). Facilitating Interprganizational Learning with Information Technology. Journal of Management Information Systems, 2(17).

Selltiz, C., Wrightsman, L. S. \& Cook, S. W. (1987). Métodos de pesquisa nas relações sociais. São Paulo: EPU.

Senge, P. (1990). The Fifth Discipline. New York: Doubleday.

Teece, D., Pisano, G. \& Shuen, A. (1997). Dynamic Capabilities and Strategic Management. Strategic Management Journal, 18, 509-533.

Ulrich, D. (1998). Capital Intelectual: Capacidad x Compromiso. Harvard Deusto Business Review, septiembre/octubre, 28-40.

Uriel, E. y Aldás, J. (2005). Análisis Multivariante Aplicado. Madrid: Thomson.

Vera, D. \& Crossan, M. (2000). Organizational Learning, Knowledge Management, and Intellectual Capital: An Integrative Conceptual Model. Working Paper.

Walsh, J. \& Ungson, G. (1991). Organizational Memory. Academy of Management Review, 1(16).

Weick, K. E. (1991). The Nontraditional Quality of Organizational Learning. Organization Science, 1(2) February, 116-123.

Zollo, M. \& Winter, S. (2002). Deliberate learning and the evolution of dynamic capabilities. Organization Science, 13(3), 339-351.

Cómo citar este capítulo:

Acosta Prado, J. C., Eiko Nakata, L., Morales Tuesca, N. A. (2017). Implicaciones de las condiciones de la gestión del conocimiento y el aprendizaje orgnizacional Sobre los resultados de la gestión un estudio comparativo. En K. Barrios Hernández, E. Olivero Vega, J. C. Acosta Prado, I. Meñaca Guerrero, A. M. Cazallo Antúnez, H. J. Medina Carrascal, ... O. Hernández Ariza, Gestión del conocimiento y capacidad de innovación. Modelos, Sistemas y Aplicaciones (pp.227-250). Barranquilla: Universidad Simón Bolívar. 